\title{
RELIABLE AMPLITUDE AND FREQUENCY ESTIMATION FOR BIASED AND NOISY SIGNALS - APPLICATION TO OSCILLATORY FAILURES DETECTION
}

\author{
T. Raïssi, D. Efimov, and A. Zolghadri \\ University of Bordeaux 1, IMS-lab, Automatic Control Group \\ 351 Cours de la Libération, Talence 33405, France
}

\begin{abstract}
Robust estimation of the amplitude, frequency and bias of unknown noisy sinusoidal signals is considered. It is only assumed that the measurements noise is bounded without any additional information such as stationarity, uncorrelation or type of distribution. The estimation problem is formulated as a Constraint Satisfaction Problem (CSP) where the amplitude, frequency and bias constitute the variables and a function relating them to the output is the constraint. Interval constraint propagation techniques are used to solve, in a reliable way, this problem. The proposed technique is applied to oscillatory failure case (OFC) detection in aircraft control surfaces. The results of simulation illustrate efficiency of the proposed technique.
\end{abstract}

\section{INTRODUCTION}

Fault detection and isolation (FDI) techniques are an increasingly important issue for many industrial systems. In this paper, the OFC related to the electronic flight control system [1] is considered. Oscillatory failure case may result in an unwanted control surface oscillation, generating unacceptably high loads or vibrations on the aircraft structure. The capability to detect these failures is very important because they have an impact on the structural design of the aircraft. Here, only OFC located in the servocontrol loop of the moving surfaces is considered $[1,2]$. The fault signals are considered to be sinusoidal with amplitude and frequency uniformly distributed over the range 1-10 Hz. A model free based technique is developed where the principle is to detect the appearance of sinousoid in a noisy signal. 
Let consider a sinusoidal signal described by

$$
y(t)=A \sin (\omega t+\phi)+A_{0}+\xi(t)
$$

where the values of the amplitude $A$, the frequency $\omega$, the offset $A_{0}$, and the phase $\phi$ are unknown and should be estimated using the measurements $y(t)$ subject to an additional signal (or a noise) $\xi$.

In the open literature, several techniques have been developed to tackle this problem [3-5]. The most known is based on least square principle and statistical analysis [6]; it is characterized by a local convergence of the estimation. In [7], this problem has been formulated as a joint nonlinear parameter and state estimation and a solution is derived using an extended Kalman filter. The main limitation of this technique is that the convergence cannot be guaranteed and it strongly depends of the initialization of the covariance matrices.

An interesting approach based on adaptive observers, with a global convergence proof in the noise-free case, has been developed in $[3,4]$. It has been shown that by defining a state variable as the time derivative of a quadratic function of the sinusoidal signal, a globally convergent estimator can be derived for reconstructing simultaneously the amplitude and the frequency of the signal. Note that the method proposed in [3] cannot be easily extended to deal with multiple amplitudes and frequencies and the performances for noisy data are not guaranteed.

In addition, the convergence could be ensured only under a persistency of excitation condition. Recently, a solution based on high-order sliding mode differentiation has been proposed in [8].

In this work, an alternative to the conventional techniques is suggested to characterize in a guaranteed way the amplitude, the frequency, and the offset of noisy signals. By guaranteed, it should be understood that not only a solution is computed, but also a domain, containing all the values of the sinus parameters that are consistent with noisy data and with the assumptions on the noise, is characterized. It is worth noting that only weak conditions on the noise properties are taken into account. Indeed, it is only assumed that the measurement noise is bounded without any additional information such as stationarity, uncorrelation, or type of distribution.

In addition, the proposed technique does not need the computation of synthesis parameters like observer gain. The estimation problem is formulated as a CSP where $A, \omega, \phi$, and $A_{0}$ constitute the variables and Eq. (1) represents the constraints for all the instants $t_{i}$. Interval constraint propagation techniques $[7,9,10]$ are used to solve in a guaranteed way this problem.

The proposed technique is applied to the aircraft OFC detection without using a physical model. 


\section{INTERVAL TECHNIQUES}

Interval techniques represent a powerful tool to tackle uncertainties propagation without any stochastic assumptions. Indeed, the evaluation of the whole set of possible model outputs could be performed using only one interval evaluation.

\section{$2.1 \quad$ Interval Operations}

A real interval $[a]=[\underline{a}, \bar{a}]$ is a connected and closed subset of $\mathbb{R}$. The set of all real intervals of $\mathbb{R}$ is denoted by $\mathbb{R}$. Real arithmetic operations are extended to intervals [11]. Let consider an operation $\circ \in\{+;-; * ; /\}$ and $[a],[b]$ two intervals, then:

$$
[a] \circ[b]=\{x \circ y \mid x \in[a], y \in[b]\} .
$$

The width of an interval $[a]$ is defined by $w[a]=\bar{a}-\underline{a}$ and its midpoint by $\operatorname{mid}[a]=(\underline{a}+\bar{a}) / 2$. The midpoint represents a punctual estimation of a variable and the radius its uncertainty. Thus, uncertainties propagation could be performed in an easy way based on interval operations.

\subsection{Inclusion Functions}

Let $\mathbf{f}: \mathbb{R}^{n} \rightarrow \mathbb{R}^{m}$; the range of the function $\mathbf{f}$ over an interval vector (called also a box) $[\mathbf{x}]$ is given by:

$$
\mathbf{f}([\mathbf{x}])=\{\mathbf{f}(\mathbf{x}) \mid \mathbf{x} \in[\mathbf{x}]\} .
$$

An interval function $[\mathbf{f}]: \mathbb{I}^{n} \rightarrow \mathbb{I}^{m}$ is an inclusion function for $\mathbf{f}$ if:

$$
\forall[\mathbf{x}] \in \mathbb{I R}^{n}, \quad \mathbf{f}([\mathbf{x}]) . \subseteq[\mathbf{f}]([\mathbf{x}]) .
$$

Usually, interval analysis is affected by overestimation, called also pessimism, which makes the evaluation of an inclusion function $[\mathbf{f}]$ larger than its range (2). Pessimism is often caused by the dependency effect, which is the lack of interval arithmetic to identify different occurrences of the same variable. For example, $x-x=0$ holds for each $x \in[x]=[-1,1]$, but $[x]-[x]$ yields $[-2,2]$.

The second source of pessimism is the wrapping effect, which appears when a complex-shaped set is wrapped into a box. An inclusion function of $\mathbf{f}$ could be obtained by replacing each occurrence of a real variable by its corresponding interval and by replacing each standard function by its interval evaluation. Such a function is called the natural inclusion function. 


\subsection{Interval Constraint Satisfaction Problems}

A CSP is defined by a set of $n$ variables $x_{1}, x_{2}, \ldots, x_{n}$ and a set of $m$ constraints $\mathcal{C}_{1}, \mathcal{C}_{2}, \ldots, \mathcal{C}_{m}$. Each variable $x_{i}$ has an initial nonempty domain $\mathcal{D}_{i}$ of possible values. Each constraint $\mathcal{C}_{i}$ involves a subset of the variables and specifies the possible combinations of values for such subset. A state of the problem is defined by an assignment of values to some or all of the variables, $x_{i}=v_{i}, \ldots, x_{j}=v_{j}$. An assignment that does not violate any constraint is called a consistent assignment. A complete assignment is one in which each variable is mentioned, and a solution to a CSP is a complete assignment that satisfies all the constraints. It is also possible to include optimizations of some objectives in CSPs to deal with constrained optimization problems. In contrast to conventional techniques, interval methods do not suffer from local convergence and the computed set is guaranteed to contain the global solutions. In addition, an empty set is returned if the CSP has no solution in the initial searching domain. The goal of propagation techniques is to reduce as much as possible the domains for the variables without losing any solution. The most known approach is based on the Waltz filtering algorithm [12] which has initially been proposed to reduce the combinatory associated with line labeling of three-dimensional scenes. The Waltz filtering is more addressed in the computer science and artificial intelligence fields [13]. Nevertheless, it has proved its efficiency in solving some control problems such as identification, filtering, and robust control [7].

Example 1. Consider a CSP defined by two variables $x$ and $y$ and three constraints:

$$
\begin{aligned}
& \left(\mathcal{C}_{1}\right): y=x^{2} \\
& \left(\mathcal{C}_{2}\right): x y=1 ; \\
& \left(\mathcal{C}_{3}\right): y=-2 x+1
\end{aligned}
$$

Assume that no prior information on the variables is available, the initial domains are thus chosen as $\left.\mathcal{D}_{i}=\right]-\infty,+\infty[$. The Waltz algorithm consists in projecting the constraints until equilibrium using interval evaluations:

$$
\begin{aligned}
& \left.\left.\left.\left(\mathcal{C}_{1}\right): y \in\right]-\infty,+\infty\right]^{2} \bigcap\right]-\infty,+\infty[=[0,+\infty[; \\
& \left.\left(\mathcal{C}_{2}\right): x \in \frac{1}{[0,+\infty[} \bigcap\right]-\infty,+\infty[=] 0,+\infty[; \\
& \left(\mathcal{C}_{3}\right): y \in(-2 *] 0,+\infty[+1) \bigcap[0,+\infty[=[0,1[; \\
& \left.x \in\left(-\frac{[0,1[}{2}+\frac{1}{2}\right) \bigcap\right] 0,+\infty\left[=\left[0, \frac{1}{2}[;\right.\right.
\end{aligned}
$$




$$
\begin{gathered}
\left(\mathcal{C}_{1}\right): y \in\left[0, \frac{1}{2}\left[^ { 2 } \bigcap \left[0,1\left[=\left[0, \frac{1}{4}[;\right.\right.\right.\right.\right. \\
\left(\mathcal{C}_{2}\right): x \in \frac{1}{[0,1 / 4[} \bigcap\left[0, \frac{1}{2}[=\emptyset ;\right. \\
y \in \frac{1}{\emptyset} \bigcap\left[0, \frac{1}{4}[=\emptyset .\right.
\end{gathered}
$$

Thus, it has been proved in two iterations that no solution exists for this CSP. In the sequel, this methodology can be used for the estimation of $A, A_{0}, \phi$ and $\omega$ in (1).

\section{SINUSOIDAL PARAMETERS ESTIMATION}

Let given a signal under the form (1) and let assume that $\xi$ is a bounded noise with a prior known bound $\bar{\xi}$. Note that this bound could be also estimated. The domain of $y$ at the time $t_{i}$ is given by

$$
\left[y\left(t_{i}\right)\right]=\left[y_{m}\left(t_{i}\right)-\bar{\xi}, y_{m}\left(t_{i}\right)+\bar{\xi}\right]
$$

where $y_{m}\left(t_{i}\right)$ is the measurement at $t_{i}$. A rigorous estimation of $A, A_{0}, \phi$, and $\omega$ consists in solving the following CSP:

$$
\left.\begin{array}{l}
y\left(t_{0}\right)=A \sin \left(\omega t_{0}+\phi\right)+A_{0} ; \\
y\left(t_{1}\right)=A \sin \left(\omega t_{1}+\phi\right)+A_{0} ; \\
\ldots \ldots \ldots \ldots \ldots \ldots \ldots \ldots \ldots \\
y\left(t_{N}\right)=A \sin \left(\omega t_{N}+\phi\right)+A_{0}
\end{array}\right\}
$$

where $A \in[A], \quad \omega \in[\omega] ; \phi \in[\phi] ;$ and $A_{0} \in\left[A_{0}\right]$.

Each constraint $i$ in (3) could be projected in two phases:

\section{Forward projection}

$$
\begin{aligned}
{\left[a_{1}\right] } & :=\left(\omega t_{i}+\phi\right) \bigcap\left[a_{1}\right] ; \\
{\left[a_{2}\right] } & :=\sin \left(\left[a_{1}\right]\right) \bigcap\left[a_{2}\right] ; \\
{\left[a_{3}\right]: } & =\left([A]\left[a_{2}\right]\right) \bigcap\left[a_{3}\right] ; \\
{\left[y\left(t_{i}\right)\right] } & :=\left(\left[a_{3}\right]+\left[A_{0}\right]\right) .
\end{aligned}
$$


The variables $a_{i}$ are introduced in order to produce simple constraints; their initial domains could be chosen as $]-\infty,+\infty[$.

The second phase consists in contracting the domain of the variables using the measurement $\left[y\left(t_{i}\right)\right]$. It is given by

\section{Backward projection}

$$
\begin{aligned}
& {\left[a_{3}\right] }:=\left(\left[y\left(t_{i}\right)\right]-\left[A_{0}\right]\right) \bigcap\left[a_{3}\right] ; \\
& {\left[A_{0}\right]:=\left(\left[y\left(t_{i}\right)\right]-\left[a_{3}\right]\right) \bigcap\left[A_{0}\right] ; } \\
& {\left[a_{2}\right]:=\left(\left[a_{3}\right] /[A]\right) \bigcap\left[a_{2}\right] ; } \\
& {[A]:=\left(\left[a_{3}\right] /\left[a_{2}\right]\right) \bigcap[A] ; } \\
& {\left[a_{1}\right]:=\arcsin \left(\left[a_{2}\right]\right) \bigcap\left[a_{1}\right] ; } \\
& {[\omega]:=\left(\frac{\left[a_{1}\right]-\phi}{t_{i}}\right) \bigcap[\omega] ; } \\
& {[\phi]:=\left(\left[a_{1}\right]-\omega t_{i}\right) \bigcap[\phi] }
\end{aligned}
$$

Usually, the reduction of the initial domain $\mathcal{D}$ is not optimal which means that it could contain inconsistent values. In such a case, a subdivision procedure is performed in order to generate two subdomains to be used again in the Forward and Backward processes. The subdivision process stops when the widths of all the generated boxes do not exceed a tolerance $\varepsilon$ chosen by the user. The algorithm 1 summarizes the estimation procedure.

Algorithm 1 generates a list $\overline{\mathcal{L}}$ of nonoverlapping boxes which is guaranteed to contain the exact solution set $\mathcal{S}$. When the tolerance $\varepsilon$ tends to 0 , the list $\overline{\mathcal{L}}$ converges to $\mathcal{S}$.

\section{FAULT DETECTION}

\subsection{Procedure}

Let assume that the only knowledge on the system is the measured outputs and the characteristics of the expected failures (OFC). Thus, the sinousoid estimation technique developed in the previous section would be useful. The idea of the fault detection scheme is to detect an abrupt frequency appearance in the 


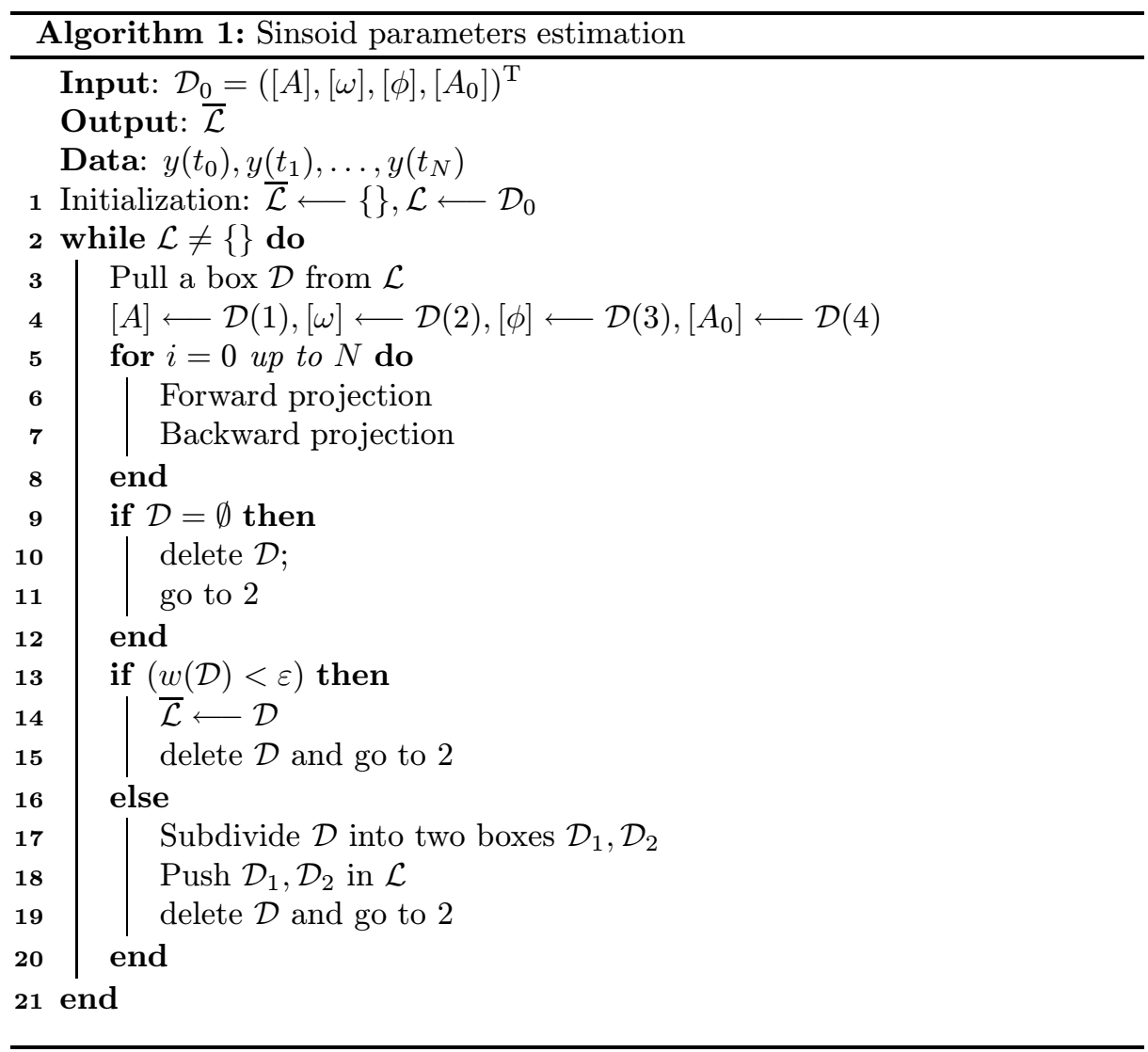

measured signal, by using a moving horizon technique, where the CSP includes only $N$ recent measurements. The horizon moves forward at each $N$ sampling times in order to include the new measurements. The horizon length should be chosen larger than a period of the sinusoid to be estimated. It is worth noting that the delay to detection is about $N T_{e}$ (where $T_{e}$ is the sampling time) when the tolerance parameter $\varepsilon$ in the algorithm 1 tends to 0 .

The methodology is summarized in algorithm 2 .

In algorithm 2, the function Convex_Hull permits to compute the smallest interval vector containing all the elements of the list $\overline{\mathcal{L}}$. This algorithm detects a fault when the domain $\mathcal{D}$ becomes nonempty. Note that in some practical cases, an abrupt frequency change could occur, two scenarios are then possible: if the frequency still belongs to the current estimated domain, then the algorithm converges to the new value. Otherwise, the algorithm converges to an empty set and it has to be reinitialized with a larger domain. 


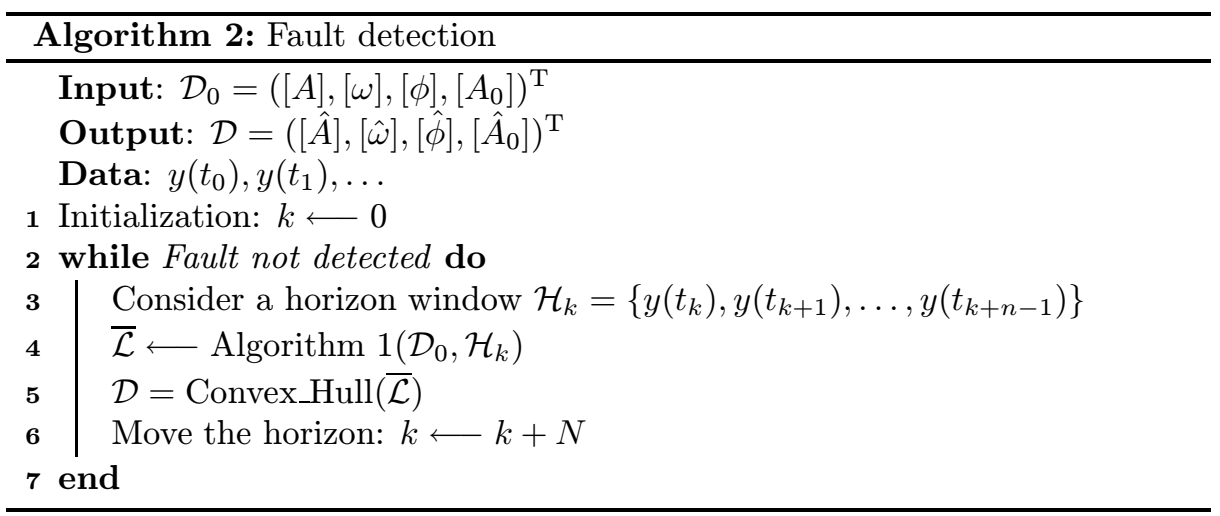

\subsection{Simulation Results}

The idea presented in the previous subsection is illustrated through the problem of the OFC related to the electronic flight control system [1]. Oscillatory failure case may result in an unwanted control surface oscillation, generating unacceptably high loads or vibrations on the aircraft structure. The capability to detect these failures is very important because they have an impact on the structural design of the aircraft. Here, only OFC located in the servocontrol loop of the moving surfaces is considered (Fig. 1) [2]. The fault signals are considered to be sinusoidal with amplitude and frequency uniformly distributed over the range $1-10 \mathrm{~Hz}$ (above $10 \mathrm{~Hz}$, the failure has no significant effects because of the lowpass ability of the actuator). The detection time is expressed in period numbers; thus, depending on the failure frequency, the time permissible for detection is varying. In $[1,2,8]$, the following nonlinear model of the hydraulic actuator is considered:

$$
\dot{y}=k_{1}(u-y) \sqrt{\frac{k_{2}}{k_{3}+k_{4}(u-y)^{2}}}
$$

where $y \in \mathbb{R}$ is the actuator position; $u \in \mathbb{R}$ is the actuator input; and $k_{i}$, $i=1, \ldots, 4$, are some positive parameters. In $[1,2]$, the case of harmonic faults is considered (with unknown frequency and magnitude) and the proposed approaches are based on nonlinear observers or Kalman filter which could be efficiently designed if the parameters $k_{i}(i=1, \ldots, 4)$ are exactly known. In the following, the present authors suggest to use the constraint satisfaction technique proposed in the previous sections which does not need the knowledge of the model (4). A faulty oscillatory signal is considered where the fault, which appears at $t=10 \mathrm{~s}$, is simulated by $f(t)=0.85 \sin (2 \pi t)$. The horizon length is taken as $N=200$ and the tolerance parameter for Algorithm 1 is $\varepsilon=0.01$. The results of simulation show that no fault is detected before $t=12 \mathrm{~s}$ and a fault is 


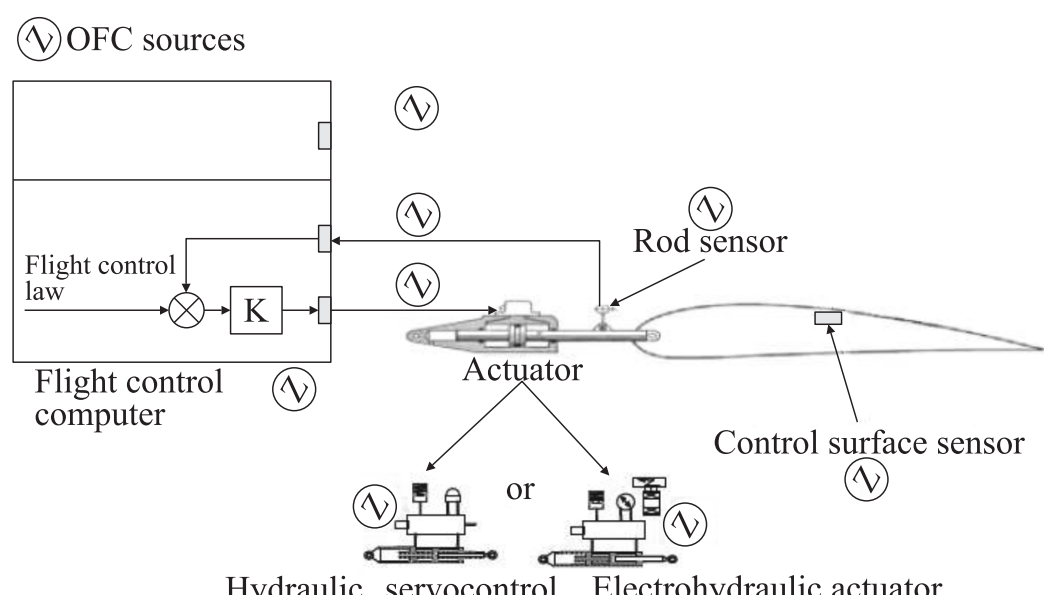

Hydraulic servocontrol Electrohydraulic actuator

Figure 1 The OFC source location in the control loop

detected over the window $t \in[12,14] \mathrm{s}$ which means that the delay to detection is about two periods. The estimated domains for the amplitude and the frequency of the fault are given by $f \in[0.9912,1.0085]$ and $A \in[0.69514,0.98926]$ and the midpoint of this domain is approximately the true value of the fault parameters. Several additional simulations give similar results for other frequencies and show that one or two periods are sufficient to detect an OFC.

\section{CONCLUDING REMARKS}

The problem of sinousoid estimation from noisy data is solved through interval consistency techniques. The proposed technique does not need a mathematical model and the accuracy of the estimates depends only on the measurement noise bound and on the partitioning parameter $\varepsilon$. The proposed sinusoid estimator is applied to the problem of oscillatory failure detection for an airplane. The results of simulations confirm robustness and performance of the proposed fault detection method. In a further work, the case of simultaneous sinusoids faults, with more parameters to be estimated, will be investigated.

\section{ACKNOWLEDGMENTS}

This work was performed in the framework of the Europeen ADDSAFE Project: Grant agreement No. FP7-233815. 


\section{REFERENCES}

1. Goupil, P. 2010. Oscillatory failure case detection in the A380 electrical flight control system by analytical redundancy. Control Eng. Practice 18(9):1110-19.

2. Alcorta Garcia, E., A. Zolghadri, and P. Goupil. 2011. A nonlinear observer-based strategy for aircraft oscillatory failure detection: A380 case study. IEEE Trans. Aerospace Electronic Syst. 47(4):2792-806.

3. Hou, M. 2005. Amplitude and frequency estimator of a sinusoid. IEEE Trans. Automatic Control 50(6):855-58.

4. Hou, M. 2007. Estimation of sinusoidal frequencies and amplitudes using adaptive identifier and observer. IEEE Trans. Automatic Control 52(3):493-99.

5. Trapero, J. R., H. Sira-Ramírez, and V.F. Batlle. 2007. An algebraic frequency estimator for a biased and noisy sinusoidal signal. Signal Process. 87(6):1188-201.

6. Stoica, P., H. B. Li, and J. Li. 2000. Amplitude estimation of sinusoidal signals: Survey, new results, and an application. IEEE Trans. Signal Process 48(2):338-52.

7. Jaulin, L., and M. Kieffer, O. Didrit, and E. Walter. 2001. Applied interval analysis. London: Springer.

8. Efimov, D., A. Zolghadri, and T. Raissi. 2011. Actuator fault detection and compensation under feedback control. Automatica 47(8):1699-705.

9. Neumaier, A. 2004. Complete search in continuous global optimization and constraint satisfaction. Acta Numerica 13:271-369.

10. Benhamou, F., and L. Granvilliers. 2006. Ch. 16 Continuous and interval constraints. In: Handbook of constraint programming. Eds. F. Rossi, P. Beek, and T. Walsh. Foundations of artificial intelligence ser. Elsevier. 2:571-603.

11. Moore, R.E. 1966. Interval analysis. Englewood Cliffs, NJ: Prentice-Hall.

12. Waltz, D. L. 1975. Generating semantic descriptions from drawings of scenes with shadows. In: The psychology of computer vision. Ed. P. H. Winston. New York: McGraw-Hill. 19-91.

13. Van Hentenryck, P. 1989. Constraint satisfaction in logic programming. Lecture Notes in Computer Science ser. MIT Press. 